\title{
Association of Lipid Metabolism Related Gene Promoter Methylation with Risk of Coronary Artery Disease
}

Wei Li ( $\square$ surlw@126.com )

Shanghai Jiao Tong University School of Medicine Affiliated Renji Hospital

\section{Yongyi Wang}

Shanghai Jiao Tong University School of Medicine Affiliated Renji Hospital

Ritai Huang

Shanghai Jiao Tong University School of Medicine Affiliated Renji Hospital

Feng Lian

Shanghai Jiao Tong University School of Medicine Affiliated Renji Hospital

\section{Genxing Xu}

Shanghai Jiao Tong University School of Medicine Affiliated Renji Hospital

\section{Weijun Wang}

Shanghai Jiao Tong University School of Medicine Affiliated Renji Hospital

\section{Song Xue}

Shanghai Jiao Tong University School of Medicine Affiliated Renji Hospital

\section{Research Article}

Keywords: coronary artery disease, lipid metabolism, methylation

Posted Date: February 22nd, 2022

DOI: https://doi.org/10.21203/rs.3.rs-1338296/v1

License: (c) (i) This work is licensed under a Creative Commons Attribution 4.0 International License.

Read Full License 
All authors declare no affiliation with the Editors, reviewers and readers of this journal as of January 2016.

${ }^{*}$ Funding Info

The present study was supported by Public Funding for Research of Cardiovascular Surgery Department, Renji Hospital.

${ }^{*}$ Conflicts of interest/Competing interests

We declare that we do not have any commercial or associative interest that represents a conflict of interest in connection with the work submitted.

\section{${ }^{*}$ Ethics statement}

This study was approved by the Medical Ethics Committee of Renji Hospital Affiliated with Shanghai Jiaotong University School of Medicine and compliant with the principles set forth by the Declaration of Helsinki.

${ }^{*}$ Consent to Participate (Ethics) -mandatory

Written Informed consent was obtained from all individual participants included in the study.

\footnotetext{
${ }^{*}$ Consent to Publish (Ethics) -mandatory
}

The Author confirms: 
- that the work described has not been published before (except in the form of an abstract or as part of a published lecture, review, or thesis);

- that it is not under consideration for publication elsewhere;

- that its publication has been approved by all co-authors, if any;

- that its publication has been approved (tacitly or explicitly) by the responsible authorities at the institution where the work is carried out.

${ }^{*}$ Authors' contributions -mandatory

All authors contributed to the study conception and design. Song Xue organized and hosted the study.Material preparation was performed by Yongyi Wang and Ritai Huang . Feng Lian and Genxing Xu undertook the laboratory experiments in this study. Data statistics was undertaken by Weijun Wang.The first draft of the manuscript was written by Wei Li and all authors commented on previous versions of the manuscript. All authors read and approved the final manuscript.

${ }^{*}$ Availability of data and material The datasets used or analysed during the current study are available from the corresponding author on reasonable request. 


\title{
Association of Lipid Metabolism Related Gene Promoter Methylation with Risk of
}

\section{Coronary Artery Disease}

Wei Li, Yongyi Wang, Ritai Huang, Feng Lian, Genxing Xu, Weijun Wang, Song Xue

Department of Cardiovascular Surgery, Renji Hospital, Shanghai Jiaotong University School of Medicine, Shanghai 200127, China.

\begin{abstract}
Background:

CAD (Coronary Artery Disease) is a complex disease that influenced by environment and genetic factors. Lipid levels are regarded as major risk factor of CAD and epigenetic mechanisms might be involved in the regulation of CAD development. This study was designed to investigate the association between DNA methylation status of 8 lipid metabolism related genes and risk of CAD in Chinese Han population.

Methods:
\end{abstract}

A total of 260 samples were used in this study, including 120 CAD cases and 140 normal health controls. DNA methylation status was tested via targeted bisulfite sequencing.

Results:

The present results indicated a significant association between hypomethylation of APOC3, CETP and APOC1 gene promoter and risk of CAD. Individuals with higher methylation levels in APOA5 and LIPC gene promoter have increased risks for CAD. In addition, a gender based breakdown analysis revealed that ANGPTL4 methylation level 
was significantly associated with CAD in male, but not in female. There were no significant differences in methylation levels of $\mathrm{APOB}$ and PCSK9 gene promoter between CAD patients and controls.

Conclusion:

Methylation status of APOC3, APOA5, LIPC, CETP and APOC1 gene promoters may be associated with development of CAD.

Key Words: coronary artery disease , lipid metabolism , methylation

\section{Introduction}

Coronary artery disease (CAD) is a common chronic inflammatory disease which has been recognized as the leading cause of death worldwide [1]. In China, it was estimated that 700,000 people died from CAD every year [2]. Blood lipid levels have been identified as important independent risk factors for CAD, including TG (Triglyceride), HDL-C (high-density lipoprotein cholesterol), LDL-C (low-density lipoprotein cholesterol) and TC (Serum total cholesterol) [3]. Recent advances have started to unravel the genetic architecture of $C A D$ and revealed that genetic variants and epigenetic regulation of lipid metabolism related genes also contribute to CAD etiology [4-6].

DNA methylation is a biological process that regulating gene expression by recruiting proteins involved in gene repression or by inhibiting the binding of transcription factors to DNA without changing the DNA sequence. Aberrant promoter hypermethylation and hypomethylation may associate with the risks of various diseases, including 
cardiovascular, cancer and metabolic diseases. Several previous studies have revealed that the methylation signatures of critical genes may play a role in CAD development [4-6]. In this study, we aim to investigate the association of methylation status in 8 lipid metabolism related genes (ANGPTL4, APOC3, APOA5, APOB, LIPC, CETP, PCSK9 and APOC1) with the risk of CAD development in Chinese Han population.

\section{Material and methods}

Study population

The participants in this study were recruited from Shanghai Renji Hospital between 2018 and 2020. A total of 120 CAD patients ( 88 male and 32 female) and 140 non-CAD controls (93 male and 47 female) were involved in this study. Criteria for CAD diagnosis were defined as followings: at least one of the major segments of coronary arteries (right coronary artery, left circumflex, or left anterior descending arteries) with more than or equal to $50 \%$ organic stenosis based on coronary angiography. All unaffected controls were determined to be free of CAD. All participants were genetically unrelated Chinese Han individuals from Shanghai. This study was approved by the Medical Ethics Committee of Renji Hospital Affiliated with Shanghai Jiaotong University School of Medicine and compliant with the principles set forth by the Declaration of Helsinki. Written informed consent was obtained from all subjects. Blood samples $(5 \mathrm{ml})$ were collected from the participants into EDTA tubes and then stored at $-80^{\circ} \mathrm{C}$ for further use.

DNA extraction, bisulfite conversion and targeted bisulfite sequencing Genomic DNA was extracted from whole blood with TianGen DNA extraction kit (TianGen 
Ltd, Beijing, China) as the manufacture instruction. DNA quality and concentration was analyzed using Electrophoresis and NanoDrop spectrophotometer (NanoDrop Technologies, Houston, TX, USA). Bisulfite conversion of 200 ng genomic DNA was performed by the EZ DNA Methylation-Gold Kit (Zymo Research, Irvine, CA, USA) according to manufacturer's standard protocol. For each gene, PCR primers were designed specifically for bisulfite-converted DNA using MethPrimer [10]. PCR primers were synthesized by Shanghai Free Biotechnology Co., Ltd (Shanghai, China). Multiplex PCR of target $\mathrm{CpG}$ regions were performed and the products were sequenced with Illumina NovaSeq sequencing instruments (Novogene, Beijing, China). All samples achieved a mean sequencing depth of $>500 \mathrm{X}$. CpG sites were named as its relative distance (in bp) to transcriptional start site (negative distances are upstream from the TSS). Methylation level of $\mathrm{CpG}$ site was calculated as the percentage of the methylated cytosines over the total tested cytosines. The average methylation level was calculated using methylation levels of all measured $\mathrm{CpG}$ sites within the gene.

Statistical Analysis

Statistical analyses were performed using the SPSS package version 21.0 (SPSS Inc, Chicago, IL, USA) and GraphPad Prism 5 Software (GraphPad Software Inc., San Diego, CA, USA). The correlation between DNA methylation and CAD was assessed using an independent sample t-test and expressed as means \pm standard deviation (SD). Both the average gene methylation data and the methylation data of the individual $\mathrm{CpG}$ loci were analyzed. Stratified analyses based on status of gender were carried out. P value $<0.05$ was considered statistically significant. 


\section{Results}

A total of 120 CAD patients and 140 healthy controls were recruited in this study. Targeted bisulfite sequencing was used to measure a total of $98 \mathrm{CpG}$ sites on 8 lipid metabolism related gene (ANGPTL4, APOC3, APOA5, APOB, LIPC, CETP, PCSK9 and APOC1) promoters. The methylation levels of each $\mathrm{CpG}$ sites were compared between patients and healthy controls (Fig 1). The methylation levels of 14 sites were significantly higher in case group than that in control group, while the methylation levels of 37 sites were significantly lower in case group than that in control group (supplementary table 1).

Figure 1: methylation level between cases and controls 

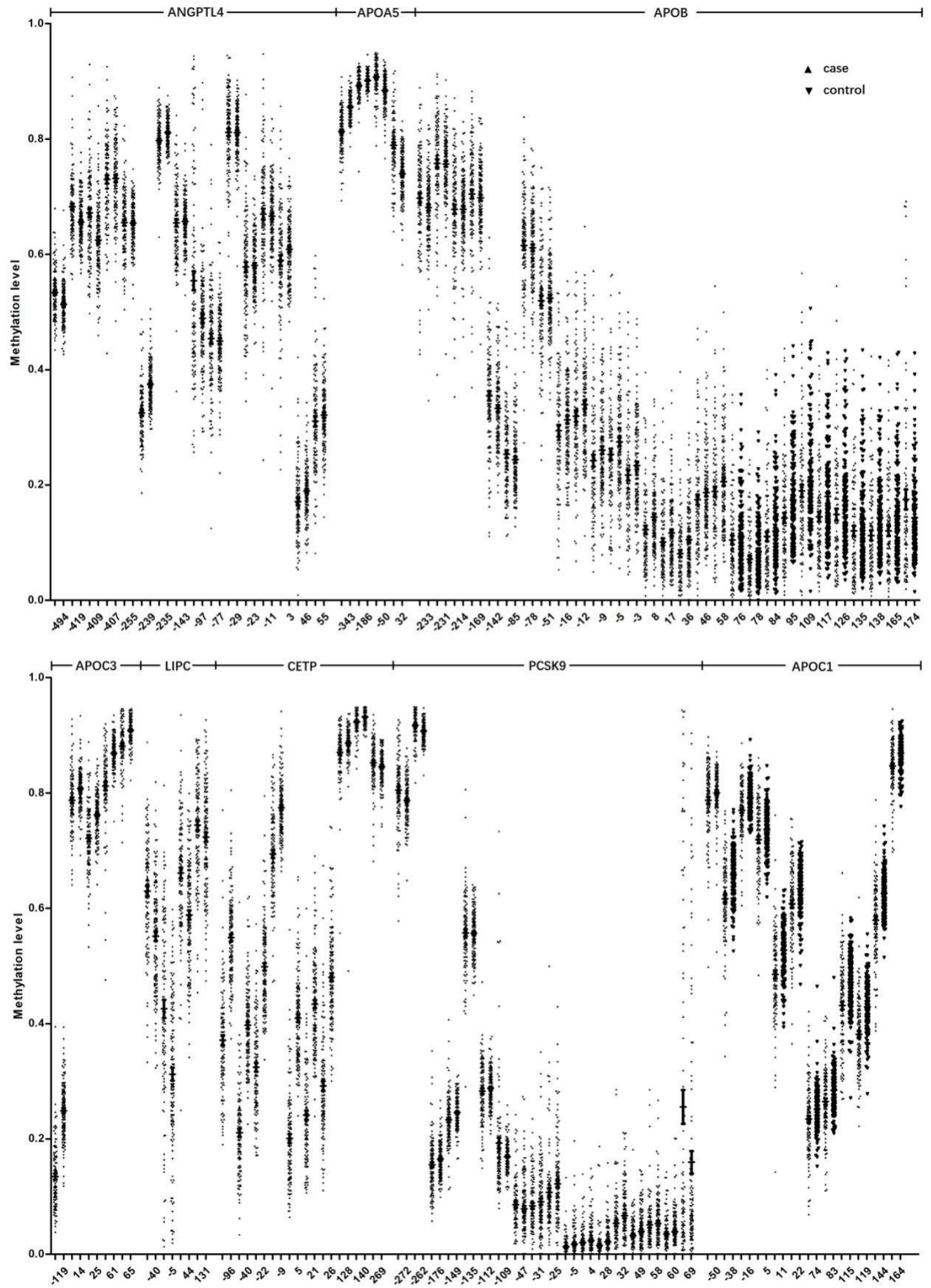

The mean methylation level of each gene was calculated (table 1). As indicated in table 1 , significantly decrease methylation levels of APOC3, CETP and APOC1 gene were observed in case group compared with control group. Most CpG sites were 
hypomethylated in APOC3 (5 of 5), CETP (9 of 10) and APOC1 (11 of 12). Significantly increase methylation levels of APOA5 and LIPC gene were observed in case group compared with control group. For LIPC, 3 of 4 CpG sites were hypermethylated. For APOA5, 2 CpG sites were hypomethylated and 2 CpG sites were hypermethylated. However, no significant correlations between methylation level and CAD were observed in ANGPTL4, APOB and PCSK9.

Table 1: Mean methylation levels (\%) of candidate genes between cases and controls

\begin{tabular}{cccc}
\hline Gene & CAD & Non-CAD & P value \\
\hline ANGPTL4 & $57.42 \pm 3.72$ & $56.93 \pm 2.68$ & 0.233 \\
APOC3 & $66.75 \pm 3.14$ & $71.84 \pm 1.99$ & $\mathbf{1 . 1 7 E - 2 6}$ \\
APOA5 & $85.07 \pm 2.03$ & $84.5 \pm 1.76$ & $\mathbf{0 . 0 2 2}$ \\
APOB & $27.78 \pm 7.06$ & $28.77 \pm 6.24$ & 0.258 \\
LIPC & $61.37 \pm 7.07$ & $54.35 \pm 6.11$ & $\mathbf{1 . 5 9 E - 1 5}$ \\
CETP & $48.7 \pm 4.58$ & $62.13 \pm 4.69$ & $\mathbf{1 . 3 3 E - 4 9}$ \\
PCSK9 & $21.46 \pm 2.48$ & $21.33 \pm 1.86$ & 0.672 \\
APOC1 & $55.95 \pm 3.94$ & $59.05 \pm 2.41$ & $\mathbf{8 . 4 0 E - 1 2}$ \\
\hline
\end{tabular}

Methylation levels (\%) are reported as means $\pm S D, P$ value less than 0.05 was in bold.

Furthermore, subgroup analyses based on gender were carried out. Gender specific analysis demonstrated that APOC3, LIPC, CETP and APOC1 remain significant in both male and female, while APOA5 remain significant only in male (table 2). ANGPTL4 was hypermethylated in male but not in female. APOB and PCSK9 methylation did not identify any significant association in stratified analysis.

Table 2: Mean methylation levels (\%) of candidate genes in male and female

\begin{tabular}{ccrrrrr}
\hline & \multicolumn{3}{c}{ Male } \\
\cline { 2 - 7 } Gene & \multicolumn{1}{c}{ CAD } & Non-CAD & \multicolumn{1}{c}{ P value } & \multicolumn{1}{c}{ CAD } & \multicolumn{1}{c}{ Non-CAD } & P value \\
\hline ANGPTL4 & $57.76 \pm 3.98$ & $56.61 \pm 2.59$ & $\mathbf{0 . 0 2 4}$ & $56.47 \pm 2.71$ & $57.55 \pm 2.76$ & 0.089 \\
APOC3 & $66.68 \pm 3.28$ & $71.67 \pm 1.98$ & $\mathbf{1 . 4 0 E - 1 8}$ & $66.96 \pm 2.79$ & $72.19 \pm 1.99$ & $\mathbf{1 . 6 2 E - 1 2}$ \\
APOA5 & $85.16 \pm 1.98$ & $84.47 \pm 1.59$ & $\mathbf{0 . 0 1 5}$ & $84.84 \pm 2.21$ & $84.55 \pm 2.07$ & 0.587
\end{tabular}




\begin{tabular}{crrrrrr} 
APOB & $27.68 \pm 7.51$ & $28.1 \pm 6.14$ & 0.697 & $28.04 \pm 5.89$ & $30.11 \pm 6.3$ & 0.168 \\
LIPC & $62.05 \pm 6.69$ & $54.01 \pm 5.78$ & $\mathbf{5 . 2 3 E - 1 5}$ & $59.55 \pm 7.82$ & $55.02 \pm 6.74$ & $\mathbf{0 . 0 0 8}$ \\
CETP & $48.3 \pm 4.38$ & $61.5 \pm 4.36$ & $\mathbf{1 . 4 4 E - 3 7}$ & $49.9 \pm 4.99$ & $63.54 \pm 5.13$ & $\mathbf{4 . 2 5 E - 1 4}$ \\
PCSK9 & $21.51 \pm 2.32$ & $21.09 \pm 1.94$ & 0.252 & $21.35 \pm 2.89$ & $21.8 \pm 1.58$ & 0.488 \\
APOC1 & $55.74 \pm 4.09$ & $58.46 \pm 2.28$ & $\mathbf{3 . 5 1 E - 0 7}$ & $56.54 \pm 3.48$ & $60.23 \pm 2.25$ & $\mathbf{7 . 9 3 E - 0 6}$ \\
\hline
\end{tabular}

Methylation levels (\%) are reported as means $\pm S D, P$ value less than 0.05 was in bold.

\section{Discussion}

CAD is a complex disease that influenced by environmental, biochemical, and genetic risk factors. Lipoprotein metabolism disorder is a causal risk factor for cardiovascular diseases in general population. DNA methylation, the most widely studied epigenetic mechanism, have an important role in the etiology of human disease. Recent studies revealed that DNA methylation changes of gene promoter might be implied in the development of CAD. In this study, we investigate the methylation status of a subset of lipid metabolism related genes in CAD patients and control subjects in Chinese Han population via targeted bisulfite sequencing.

APOC3 encodes a protein component of triglyceride (TG)-rich lipoproteins (TRLs) and play a role in promoting the hepatic secretion of TRLs. APOC3 was an inhibitor of lipoprotein lipase (LPL) enzyme activity and preventing TRLs clearance [11, 12]. Loss-of-function APOC3 mutations were associated with low plasma triglyceride levels and reduced risk of cardiovascular disease $[13,14]$. Genetic variation in the promoter region APOC3 gene was associated with an increased risk of hypertriglyceridemia, metabolic syndrome and CAD [15-17]. Overexpression of APOC3 gene in transgenic animal induces severe hypertriglyceridemia, while APOC3 gene deletion results in hypotriglyceridemia [18-21]. In this study, APOC3 gene was hypomethylated in CAD 
patients. The methylation level of CpG -119 in APOC3 gene promoter was significantly lower in CAD group than that in control group (13.5\% VS $24.5 \%)$, and might lead to higher gene expression.

APOC1 gene encodes a member of the apolipoprotein C1 family and resides within APOE/APOC1/APOC2 gene cluster. This gene was predominantly expressed in the liver, lung, skin, spleen, adipose tissue, and brain [22]. APOC1 plays an important role in high density lipoprotein (HDL) and very low density lipoprotein (VLDL) metabolism. APOC1 was a very potent and highly selective inhibitor of cholesteryl ester transfer protein (CETP) in plasma [23, 24]. Transgenic analysis revealed that increased expression of APOC1 inhibit the hepatic uptake of lipoproteins and results in combined hyperlipidemia [25-27]. In this study, $12 \mathrm{CpG}$ sites in APOC1 were analyzed and $11 \mathrm{CpG}$ sites from -38 to +164 were hypomethylated in CAD patients. The methylation level of CpG -50 between case and control group did not reach statistically significant.

Cholesteryl ester transfer protein (CETP) mediates the transfer of cholesteryl ester from high density lipoprotein (HDL) to other lipoproteins, and promotes the formation of TG-rich and CE-poor HDL particles. It has been reported that genetic variation in CETP was associated with HDL-C levels [28-30]. CETP deficiency was associated with a slow progression of CAD, high HDL-C, low HDL-TG levels and a higher HDL particle size [31, 32]. CETP inhibitors effectively reduce LDL-C and increase HDL-C levels and may be effective in reducing atherosclerosis and cardiovascular events [33]. In this study, 9 of 10 CpG sites from -94 to +140 in CETP gene were hypomethylated in CAD patients. The methylation level of CpG +269 between case and control group did not reach statistically 
significant.

LIPC encodes hepatic triglyceride lipase, which participates in the hydrolysis of triglycerides (TGs) and phospholipids, is mainly expressed and secreted from liver. Variants in the promoter region of LIPC were reported to be correlated with high HDL-C levels $[34,35]$. It has been reported that in familial hypercholesterolemia, subjects with previous history CAD had higher LIPC DNA methylation levels compared with that without CAD history [36]. Our study revealed 3 of $4 \mathrm{CpG}$ sites from -40 to +44 in LIPC gene were hypermethylated in CAD patients. The methylation level of $\mathrm{CpG}+131$ between case and control group did not reach statistically significant.

APOA5 gene plays an important part in the regulation of blood triglyceride levels and regarded as a major risk factor for coronary heart disease. Genome-wide methylation analysis found APOA5 was hypomethylated in obesity children [37]. APOA5 hypomethylation was also involved in aortic valve stenosis (AVS) [38]. Genetic variation of APOA5 gene was associated with the levels of plasma lipid and increased risk of cardiovascular disease $[39,40]$. The mean methylation level of APOA5 gene was relatively high in CAD patients than in control group. However, CpG -50 and $C p G+32$ were hypermethylated while CpG -343 and CpG -186 were hypomethylated.

In conclusion, targeted bisulfite sequencing was used to assess the methylation status of 8 lipid metabolism related candidate genes in patients diagnosed with CAD and control subjects without CAD. We revealed that methylation levels of APOC3, CETP and APOC1 gene promoters were relatively lower in CAD group than that in control group. Methylation levels of APOA5 and LIPC gene promoters were higher in CAD group than that in control 
group. Our findings indicating the hypothesis that DNA methylation in lipid related genes plays a role in the development of $C A D$, and may provide some new implications for prevention and treatment of CAD. However, there are some limitations in this study. The expression levels of target genes were not investigated, so we cannot determine whether promoter methylation affect gene expression. Moreover, Variations in the gene region and DNA methylation might have duel effect on disease development. Further study of larger sample size with expression and genotyping data is needed to confirm our results.

\section{Acknowledgement}

We thank Hu Liu from Shanghai Lehao Bio-Science Company for his technical support of gene sequencing.

\section{References:}

[1] Roger VL, Go AS, Lloyd-Jones DM, et al. Heart disease and stroke statistics--2011 update: a report from the American Heart Association. Circulation. 2011; 123(4):e18-e209.

[2] Wang F, Xu CQ, He Q, et al. Genome-wide association identifies a susceptibility locus for coronary artery disease in the Chinese Han population. Nature Genetics. 2011; 43(4):345-349.

[3] Foody J, Yong H, Ji L, et al. Unique and Varied Contributions of Traditional CVD Risk Factors: A Systematic Literature Review of CAD Risk Factors in China. Clin Med Insights Cardiol. 2013; 2013(7):59-86.

[4] Duan L, Liu C, Hu J , et al. Epigenetic mechanisms in coronary artery disease: The current state and prospects. Trends Cardiovasc Med. 2018;28(5):311-319. 
[5] Pjanic M, Miller CL, Wirka R, et al. Genetics and Genomics of Coronary Artery Disease. Current Cardiology Reports. 2016;18(10):102.

[6] Musunuru K, Kathiresan S. Genetics of Common, Complex Coronary Artery Disease. Cell. 2019;177(1):132-145.

[7] Ghaznavi H, Mahmoodi K, Soltanpour MS. A preliminary study of the association between the ABCA1 gene promoter DNA methylation and coronary artery disease risk. Molecular Biology Research Communications. 2018; 7(2):59-65.

[8] Su J, Li J, Yu Q, et al. Association of PON1 gene promoter DNA methylation with the risk of Clopidogrel resistance in patients with coronary artery disease. Journal of clinical laboratory analysis, 2019; 33(5):e22867.

[9] Zhuang J, Peng W, Li H, et al. Methylation of p15INK4b and Expression of ANRIL on Chromosome 9p21 Are Associated with Coronary Artery Disease. Plos One. 2012; 7(10):e47193.

[10] Li LC, Dahiya R. Methprimer: Designing Primers for Methylation PCRs. Bioinformatics. 2002; 18(11):1427-1431.

[11] Taskinen MR, Packard CJ, Boren J. Emerging Evidence that ApoC-III Inhibitors Provide Novel Options to Reduce the Residual CVD. Curr Atheroscler Rep. 2019, 21(8):27.

[12] Akoumianakis I, Zvintzou E, Kypreos K, et al. ANGPTL3 and Apolipoprotein C-III as Novel Lipid-Lowering Targets. Current Atherosclerosis Reports. 2021; 23(5):20.

[13] Jorgensen AB, Frikke-Schmidt R, Nordestgaard BG, et al. Loss-of-Function Mutations in APOC3 and Risk of Ischemic Vascular Disease. N Engl J Med. 2014; 3711(1):32-41.

[14] Crosby J, Peloso GM, Auer PL, et al. Loss-of-function mutations in APOC3, triglycerides, and coronary disease. N Engl J Med. 2014; 371(1):22-31. 
[15] Li WW, Dammerman MM, Smith J, et al. Common genetic variation in the promoter of the human apo CIII gene abolishes regulation by insulin and may contribute to hypertriglyceridemia. Journal of Clinical Investigation. 1996; 96(6):2601-2605.

[16] Pollex RL, Ban MR, Young TK, et al. Association between the $-455 \mathrm{~T}>\mathrm{C}$ promoter polymorphism of the APOC3 gene and the metabolic syndrome in a multi-ethnic sample. BMC Med Genet. 2007; 8(80): 1471-2350 .

[17] Olivieri O, Stranieri C, Bassi A, et al. ApoC-III gene polymorphisms and risk of coronary artery disease[J]. Journal of Lipid Research, 2002, 43(9):1450-1457.

[18] Jong MC, Rensen PC, Dahlmans VE, et al. Apolipoprotein C-III deficiency accelerates triglyceride hydrolysis by lipoprotein lipase in wild-type and apoE knockout mice. Journal of Lipid Research. 2001; 42(10):1578-1585.

[19] Yan H, Niimi M, Matsuhisa F, et al. Apolipoprotein CIII Deficiency Protects Against Atherosclerosis in Knockout Rabbits. Arteriosclerosis Thrombosis and Vascular Biology. 2020; 40(9).

[20] Ding Y, Wang Y, Hong Z, et al. Hypertriglyceridemia and delayed clearance of fat load in transgenic rabbits expressing human apolipoprotein CIII. Transgenic Research. 2011; 20(4):867. [21] Ito Y, Azrolan N, O'Connell A, et al. Hypertriglyceridemia as a result of human apo CIII gene expression in transgenic mice. Science. 1990; 249(4970):790-793.

[22] Lauer SJ, Walker D, Elshourbagy NA, et al. Two copies of the human apolipoprotein C-I gene are linked closely to the apolipoprotein E gene. Journal of Biological Chemistry. 1988; 263(15):7277-7286.

[23] Gautier T, Masson D, Jong MC, et al. Apolipoprotein CI Deficiency Markedly Augments 
Plasma Lipoprotein Changes Mediated by Human Cholesteryl Ester Transfer Protein (CETP) in CETP Transgenic/ApoCI-knocked Out Mice. Journal of Biological Chemistry. 2002;

277(35):31354.

[24] Gautier T, Masson D, de Barros JP, et al. Human Apolipoprotein C-I Accounts for the Ability of Plasma High Density Lipoproteins to Inhibit the Cholesteryl Ester Transfer Protein Activity. Journal of Biological Chemistry. 2000; 275(48):37504-37509.

[25] Shachter NS, Ebara T, Ramakrishnan R, et al. Combined hyperlipidemia in transgenic mice overexpressing human apolipoprotein Cl. Journal of Clinical Investigation.1996; 98(3):846-855. [26] Jong MC, Dahlmans VE, Gorp P, et al. In the absence of the low density lipoprotein receptor, human apolipoprotein $\mathrm{C}$ 1 overexpression in transgenic mice inhibits the hepatic uptake of very low density lipoproteins via a receptor-associated protein-sensitive pathway. The Journal of clinical investigation. 1996; 98(10):2259-67.

[27] Berbee JF, Hoogt CC, Sundararaman D, et al. Severe hypertriglyceridemia in human APOC1 transgenic mice is caused by apoC-I-induced inhibition of LPL. Journal of Lipid Research, 2005, 46(2):297-306.

[28] Takahashi K, Jiang XC, Sakai N, et al. A missense mutation in the cholesteryl ester transfer protein gene with possible dominant effects on plasma high density lipoproteins. Journal of Clinical Investigation.1993; 92(4):2060-2064.

[29] Arai T, Yamashita S, Sakai N, et al. A novel nonsense mutation (G181X) in the human cholesteryl ester transfer protein gene in Japanese hyperalphalipoproteinemic subjects. Journal of Lipid Research. 1996; 37(10):2145.

[30] Boekholdt SM, Thompson JF. Natural genetic variation as a tool in understanding the role of 
CETP in lipid levels and disease. Journal of Lipid Research. 2003; 44(6):1080-1093.

[31] Inazu A, Jiang XC, Haraki T, et al. Genetic cholesteryl ester transfer protein deficiency caused by two prevalent mutations as a major determinant of increased levels of high density lipoprotein cholesterol. J Clin Invest. 1994; 94(5): 1872-1882.

[32] Arai T, Tsukada T, Murase T, et al. Particle size analysis of high density lipoproteins in patients with genetic cholesteryl ester transfer protein deficiency. Clinica Chimica Acta. 2000; 301(1-2):103-117.

[33] Mabuchi H, Nohara A, Inazu A. Cholesteryl Ester Transfer Protein (CETP) Deficiency and CETP Inhibitors. Molecules \& Cells. 2014; 37(11):777-784.

[34] Aaron I, Sayed-Tabatabaei FA, jajou OT, et al. The -514 C->T hepatic lipase promoter region polymorphism and plasma lipids: a meta-analysis. Journal of Clinical Endocrinology \& Metabolism. 2004(8):3858-3863.

[35] Hodoglugil U, Williamson DW, Mahley RW. Polymorphisms in the hepatic lipase gene affect plasma HDL-cholesterol levels in a Turkish population. Journal of Lipid Research. 2010; 51(2):422.

[36] Guay SP, Brisson D, Lamarche B, et al. Epipolymorphisms within lipoprotein genes contribute independently to plasma lipid levels in familial hypercholesterolemia[J]. Epigenetics, 2014, 9(5):718-729.

[37] Li Y, Zhou Y, Zhu L, et al. Genome-wide analysis reveals that altered methylation in specific CpG loci is associated with childhood obesity. Journal of Cellular Biochemistry, 2018, 119(9): 7490-7497.

[38] Radhakrishna U, Albayrak S, Alpay-Savasan Z, et al. Genome-Wide DNA Methylation 
Analysis and Epigenetic Variations Associated with Congenital Aortic Valve Stenosis (AVS). Plos One.2016; 11(5):e0154010.

[39] Zhao T, Zhao J. Association of the apolipoprotein A5 gene -1131 T $>$ C polymorphism with fasting blood lipids: A meta-analysis in 37859 subjects. BMC Medical Genetics. 2010; 11:120.

[40] Wang J, Ban MR, Kennedy BA, et al. APOA5 genetic variants are markers for classic hyperlipoproteinemia phenotypes and hypertriglyceridemia. Nature Clinical Practice Cardiovascular Medicine. 2008; 5(11):730-737.

Supplementary table 1: methylation levels (\%) of CpG sites between cases and controls 


\section{Supplementary Files}

This is a list of supplementary files associated with this preprint. Click to download.

- Supplementarytable1.pdf 